\title{
Beneficial effects of probiotic bacteria isolated from breast milk
}

\author{
Federico Lara-Villoslada ${ }^{1}$, Mónica Olivares ${ }^{1}$, Saleta Sierra ${ }^{1}$, Juan Miguel Rodríguez ${ }^{2}$, Julio Boza ${ }^{1}$ \\ and Jordi Xaus ${ }^{1 *}$ \\ ${ }^{1}$ Nutrition and Health Department, Puleva Biotech. Cno, De Purchil no 66, 18004, Granada (Spain) \\ ${ }^{2}$ Nutrition and Bromatology Department, Universidad Complutense, Ciudad Universitaria, 28040 Madrid (Spain)
}

Breast milk is the best food for the neonate because it provides a unique combination of proteins, carbohydrates, lipids, minerals and vitamins that ensures the correct growth and development of the infant. In addition, it also contains bioactive compounds responsible for a wide range of beneficial effects such as the promotion of immune system maturation and the protection against infections. Among these bioactive agents, probiotic bacteria have been recently isolated from human milk. The present work reviews the beneficial effects of these bacteria both in animal models and in clinical trials. The promotion of immune system maturation and defence against infections as well as the anti-inflammatory properties are among the main healthy effects of these bacteria. The isolation of probiotic bacteria with beneficial effects for the host provides scientific support for the supplementation of infant formula with these bacteria, in order to advance the pursuit of the main goal of formula: to mimic breast milk and its functional effects as closely as possible.

Probiotic: Human milk: Infant nutrition: Immune system

Human milk is a complex species-specific biological fluid adapted to perfectly satisfy the nutritional and immunological needs of the neonate. It has been demonstrated that breast milk confers protection against different infectious diseases since the incidence of these disorders is lower in breast-fed than in formula-fed infants ${ }^{1,2}$. It has been suggested that this antiinfective effect is due to several bioactive compounds present in colostrum and/or in mature milk. These include immunoglobulins, immune cells, antimicrobial acids, polyamines, oligosaccharides, lysosyme, glycoproteins such as lactoferrin and bioactive peptides, which, acting individually or synergistically, could inhibit pathogenic microorganisms 3,4

Recent studies have demonstrated that human milk, far from being a sterile fluid, constitutes an excellent and continuous source of commensal bacteria for the infant gut $^{5,6}$. These bacteria could also play an important role in the reduction of incidence and severity of infectious diseases in breastfed children. This hypothesis is supported by relatively old studies reporting the loss of antimicrobial activity in pasteurised human milk ${ }^{7}$.

Among the bacteria found in human milk, those belonging to the species Staphilococcus, Lactococcus, Enterococcus and Lactobacillus are the most frequent ${ }^{5,6}$ (Table 1). There is increasing interest in some of these breast milk lactobacilli, such as L. gasseri, L. salivarius, L. rhamnosus, L. plantarum and L. fermentum, because they are considered as potentially probiotic species (Table 1 ).

\section{Breastfeeding and protection against diseases}

In developing countries, one of the main causes of death in the paediatric age group is the infectious disease, specially gastroenterocolitis and respiratory infections. Newborns who have not been breastfed show a 17-fold higher risk of being hospitalised due to pneumonia than those who exclusively received human milk ${ }^{8}$. Similarly, the risk of death due to diarrhoea increases 14.2-fold in weaned infants ${ }^{9}$. Breastfeeding has also been related to a lower incidence of acute otitis media ${ }^{10}$, urinary tract infection ${ }^{11}$ and meningitis caused by Haemophilus influenzae $e^{12}$.

Besides its anti-infective properties, it has been demonstrated that human milk modulates the immune system of the newborn ${ }^{13}$. Although an anti-inflammatory activity has not yet been demonstrated in vivo, several epidemiologic studies suggest that breast-fed children are protected against infections without the observation of evident lesion of the intestinal or respiratory mucosa due to an inflammatory response ${ }^{14}$. This is probably the result of an anti-inflammatory system better regulated by bioactive components of human milk.

The immunomodulatory action of breast milk could also explain the better antibody production response in breast-fed compared to formula-fed infants after vaccination against poliomyelitis, tetanus and diphtheria ${ }^{15}$.

Neonates who received breast milk also have a more favourable intestinal microbiota than those fed infant formula ${ }^{16}$, which is probably due to presence of lactic acid bacteria in human milk, besides other bifidogenic compounds such as oligosaccharides ${ }^{17}$. It has been suggested that these differences in intestinal microbiota could be responsible for some of the beneficial effects seen in breast-fed infants. It has been known for several decades that lactobacilli and bifidobacteria inhibit the growth of pathogen microorganisms such as Staphylococcus aureus, Salmonella typhimurium, 
Table 1. Bacterial species generally isolated from the breast milk of healthy women

\begin{tabular}{ll}
\hline Bacterial group & Main species \\
\hline Staphylococcus spp. & S. epidermidis \\
& S. hominis \\
S. capitis \\
S. aureus \\
Streptococcus spp. & S. salivarius \\
& S. mitis \\
S. parasanguis \\
S. peores \\
L. gasseri \\
L. rhamnosus \\
L. acidophilus \\
L. plantarum \\
L. fermentum \\
L. salivarius \\
L. reuteri \\
Enterococcus sp. & E. faecium \\
& E. faecalis \\
\hline
\end{tabular}

Modified from Martin et al. ${ }^{32}$.

Yersinia enterocolitica and Clostridium perfringens ${ }^{18}$. These bacteria competitively colonise the intestine of the child, thus preventing the adhesion of pathogens. Moreover, a competition for nutrients is established and this is another mechanism that inhibits the growth of pathogenic microorganisms ${ }^{19}$.

Intestinal colonisation by commensal bacteria also plays a key role in the maintenance of immune system homeostasis. These bacteria stimulate $T_{\mathrm{H}} 1$ responses and compensate the trend towards $\mathrm{T}_{\mathrm{H}} 2$ responses characteristic of the neonatal immune system. It has been reported that the administration of specific probiotics to newborns reduces the incidence of atopic manifestations $^{20}$ and also of inflammatory processes where a $\mathrm{T}_{\mathrm{H}} 2$ response is involved, such as necrotizing enterocolitis ${ }^{21}$.

\section{Probiotics isolated from breast milk}

The description of the presence of bacteria in human milk dates back 30 years, but then it was assumed to be a contamination occurring during sample extraction ${ }^{22}$. At the beginning of the 21 st century, two European groups independently demonstrated the presence of lactic acid bacteria in human milk and their probiotic potential ${ }^{5,6}$. Thus Heikkilä et al. reported that these human milk bacteria protect both mother and newborn from infections caused by Staphilococcus aureus ${ }^{5}$. In a similar way and in a series of reports, Martín et al. ${ }^{6}$ described the isolation of lactic acid bacteria from human milk, namely, L. gasseri CECT5714 ${ }^{23}$, L. salivarius CECT5713 ${ }^{24}$ and L. fermentum CECT5716 ${ }^{23}$. Besides these strains, there are other commercial strains related to human milk. One of them is $L$. reuteri ATCC55730, which is claimed to be derived from human milk but its origin has not been published yet. L. rhamnosus LGG, although originally isolated from intestinal sources ${ }^{25}$, has also been found in human milk by the Finnish group ${ }^{5}$. Nevertheless, this work focuses on the beneficial effects of $L$. gasseri CECT5714, L. salivarius CECT5713 and L. fermentum CECT5714, which have been summarised in Table 2.

\section{Anti-microbial effects}

Protection against viral or bacterial infections is one of the most frequent claims made for probiotic consumption. Different mechanisms have been suggested to explain this anti-microbial activity (Fig. 1). In vitro studies demonstrate that certain probiotic strains produce anti-microbial compounds, such as organic acids, $\mathrm{H}_{2} \mathrm{O}_{2}$ and/or bacteriocins ${ }^{26}$, that have been reported to inhibit the growth of E. coli, Salmonella spp. and Listeria monocytogenes ${ }^{27}$. No bacteriocin-producing lactobacillus has been found in human milk, although a high production of $\mathrm{H}_{2} \mathrm{O}_{2}$ has been reported ${ }^{23}$.

Table 2. Beneficial effects of some breast milk-isolated probiotic strains

\begin{tabular}{|c|c|c|}
\hline Strain & Beneficial effect & Reference \\
\hline \multirow[t]{6}{*}{ L. salivarius CECT5713 } & Intestinal colonisation & Martin et al., $2006^{24}$ \\
\hline & Production of antimicrobial compounds & Martin et al., $2006^{24}$ \\
\hline & No D-lactic production & Martin et al., $2006^{24}$ \\
\hline & Anti-microbial effect & Olivares et al., $2006^{27}$ \\
\hline & Immunomodulatory effect & Díaz-Ropero et al., $2006^{30}$ \\
\hline & Anti-inflammatory effect & Peran et al., $2005^{37}$ \\
\hline \multirow[t]{9}{*}{ L. gasseri CECT5714 } & Intestinal colonisation & Martin et al., $2005^{23}$ \\
\hline & & Olivares et al., $2006^{38}$ \\
\hline & Improved gastrointestinal function & Lara-Villoslada et al., $2007^{39}$ \\
\hline & & Olivares et al., $2006^{38}$ \\
\hline & Production of antimicrobial compounds & Martin et al., $2005^{23}$ \\
\hline & Anti-microbial effect & Olivares et al., $2006^{27}$ \\
\hline & Immunomodulatory effect & Olivares et al., $2006^{35}$ \\
\hline & & Lara-Villoslada et al., $2007^{39}$ \\
\hline & Anti-allergic effect & Olivares et al., $2005^{33}$ \\
\hline \multirow[t]{9}{*}{ L. fermentum CECT5716 } & Intestinal colonisation & Martin et al., $2005^{23}$ \\
\hline & & Olivares et al., $2006^{36}$ \\
\hline & Production of antimicrobial compounds & Martin et al., $2005^{23}$ \\
\hline & Anti-microbial effect & Olivares et al., $2006^{27}$ \\
\hline & Immunomodulatory effect & Diaz-Ropero et al., $2006^{30}$ \\
\hline & Enhanced effects of vaccination & Olivares et al., $2007^{36}$ \\
\hline & Anti-inflammatory effect & Diaz-ropero et al., $2006^{30}$ \\
\hline & & Peran et al., $2005^{37}$ \\
\hline & & Peran et al., $2007^{34}$ \\
\hline
\end{tabular}




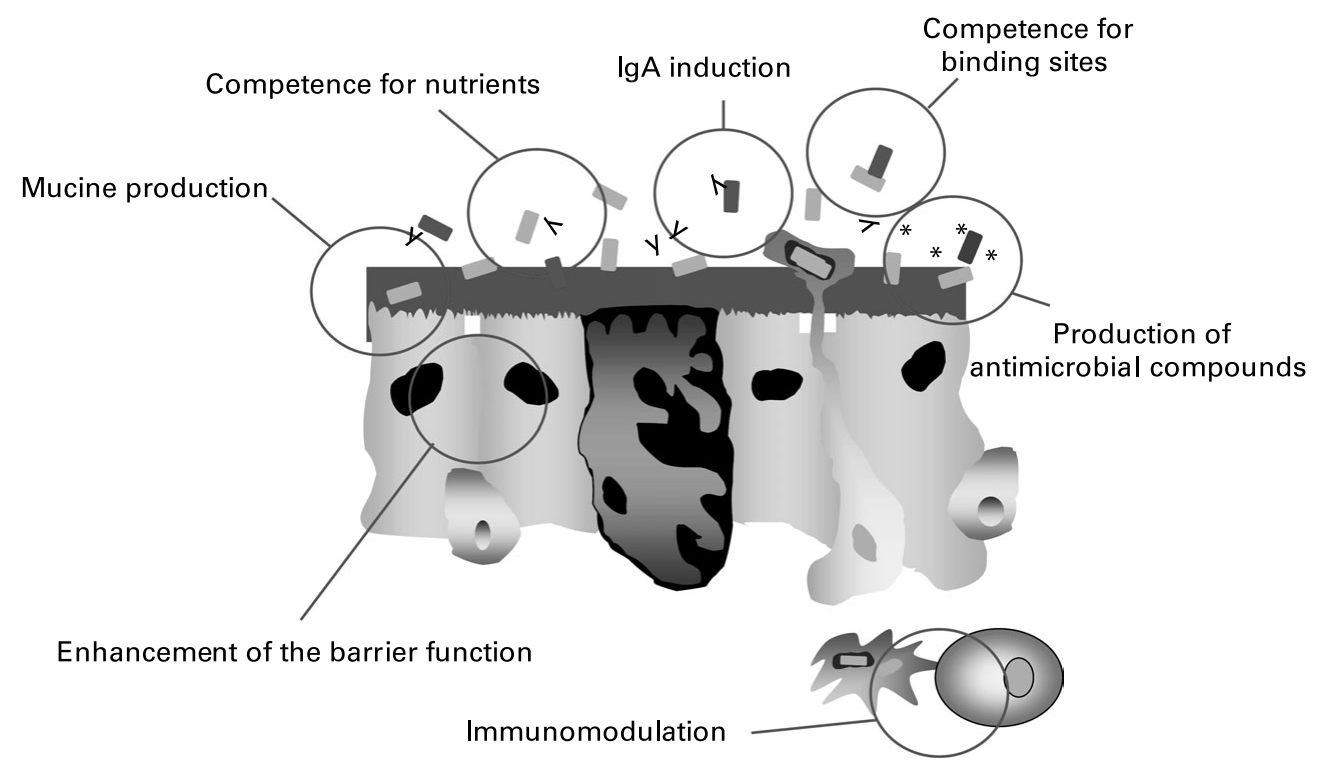

Fig. 1. Intestinal anti-infective mechanisms of probiotic bacteria.

In addition, those strains belonging to the species $L$. reuteri produce reuterin, another antimicrobial compound ${ }^{28}$. It has also been shown that some bacteria present in human milk improve the intestinal barrier function by increasing mucine production and reducing intestinal permeability ${ }^{27}$. However, competition with entero-toxigenic bacteria for nutrients and for epithelial intestinal cell receptor binding sites is probably the main anti-infective mechanism of probiotic bacteria $^{26,27}$ (Fig. 1).

The human milk-isolated probiotics L. gasseri CECT5714, L. salivarius CECT5713 and L. fermentum CECT5714 have been reported to inhibit the adhesion of Salmonella cholerasuis to mucins and to increase the survival of mice infected with this pathogen ${ }^{27}$. It was demonstrated that the protective effect of L. salivarius CECT5713 is significantly higher than the effect of a reuterin producing strain ${ }^{29}$. This is probably due to the combination of the immunomodulatory role and the competitive activity reported for $L$. salivarius CECT5713 $3^{30}$.

Different clinical trials have demonstrated that, when breastfeeding is not possible, infant formula supplemented with probiotics protect children from infectious diseases. To our knowledge, most of the studies have involved supplementation with L. rhamnosus LGG, which have demonstrated a reduction in the incidence of rotavirus infection and in the duration of diarrhoea ${ }^{31}$. Currently, clinical studies are in progress to evaluate the tolerance and effectiveness of other breast milk strains, such as L. reuteri ATCC55730 and L. salivarius CECT5713.

\section{Immunomodulatory properties}

Intestinal colonisation is often the result of the first contact of the newborn with microorganisms, which is crucial for the development of the immune system of the neonate. It has been reported that differences in the composition of intestinal microbiota influence the incidence of certain pathologies with an important immunological component, such as allergic or inflammatory processes ${ }^{32}$. The anti-allergic effect of probiotics could be explained on the basis of the Hygiene Hypothesis and the $T_{H} 1 / T_{H} 2$ balance. Probiotics induce a $T_{H} 1$ response, and thus down-regulate the production of $\mathrm{T}_{\mathrm{H}} 2$ cytokines, responsible for the allergic response.

In contrast, the anti-inflammatory effect of probiotics is more difficult to explain. In vitro studies have demonstrated that the immunomodulatory effects of probiotics depend on the cell environment. Thus, in the absence of additional stimulus, the breast milk probiotics L. salivarius CECT5713 and L. fermentum CECT5716 enhance the production of $\mathrm{T}_{\mathrm{H}} 1$ cytokines such as IL-2 and IL-12 and the inflammatory mediator $\mathrm{TNF}-\alpha^{30}$. However, when cells are incubated in the presence of lipopolysaccharide, together with the probiotics, a reduction of $\mathrm{T}_{\mathrm{H}} 1$ cytokines is observed ${ }^{30}$. This regulatory mechanism is probably based on the production of IL-10, an immunosuppressive cytokine, which has been reported to be increased by these probiotic strains ${ }^{30}$.

The immunomodulatory effects of probiotics have also been reported in animal models of pathologies where the immune system is involved. Different probiotic strains isolated from human milk have been reported to enhance the immune defence of mice, increasing both natural and acquired immune responses ${ }^{30}$. This immune-stimulating activity could be also involved in the anti-infective role previously mentioned for these bacteria in an animal model of Salmonella infection ${ }^{27}$. In addition, the breast milk probiotic L. gasseri CECT5714 in combination with L. coryniformis CECT5711 reduces the incidence and severity of the allergic response in an animal model of cow's milk protein allergy $^{33}$. In a recent report, L. fermentum CECT5716 showed a beneficial effect in an animal model of intestinal inflammation, reducing the inflammatory response and the intestinal damage ${ }^{34}$.

Probiotics have also been reported to modulate the immune response of healthy humans, as shown by a recent study which reports an increase in phagocytic activity, in the number of natural killer cells and in the plasma concentration of $\operatorname{IgA}$ in healthy humans consuming human milk-isolated probiotics daily for 3 months ${ }^{35}$. A more recent report demonstrates that the consumption of L. fermentum CECT5716 enhances the response to 
influenza vaccination in healthy volunteers aged 26-40 and reduces the incidence of influenza-like illness ${ }^{36}$.

In addition, the beneficial effect of probiotics in allergic processes has been widely reported. In this sense, the consumption of probiotics present in human milk, especially L. rhamnosus LGG, has been shown to reduce the incidence and severity of atopic dermatitis in children ${ }^{20}$. Although less is known about other allergic disorders, there is data to support a positive effect of L. gasseri CECT5714 in adults with respiratory allergy ${ }^{33}$.

\section{Gastrointestinal benefits}

There is increasing interest in the manipulation of intestinal microbiota with the aim of improving gastrointestinal function and nutrient absorption. Different reports demonstrate that human milk probiotics colonise the intestine and increase faecal lactobacilli counts thus modifying intestinal microbiota both in rodents $^{37}$ and humans ${ }^{38}$. In addition, molecular analysis show that these bacteria are metabolically active in the human gut, increasing the production of functional metabolites such as butyrate $^{38}$, which is the main energy source for colonocytes and plays a key role in the modulation of intestinal function. In the previously mentioned clinical trial ${ }^{38}$, an increase in faecal moisture, and in stool frequency and volume was observed which could be related to the increase in the faecal concentration of butyric acid.

Similarly, the administration of $L$. gasseri CECT5714 also caused an increase in faecal lactobacilli counts in a clinical trial in children aged $3-12^{39}$. In the same study the cytotoxicity of the faecal water of children who received the probiotic has been shown to be lower than that of the control children ${ }^{39}$. Finally, in another clinical trial the supplementation of infant formulas with L. rhamnosus LGG has been demonstrated to improve neonate growth pattern, which could suggest an increased bioavailability of nutrients in these infants ${ }^{40}$.

\section{Conclusions}

Breastfeeding is the main determinant of the intestinal colonisation of the neonate, which, apart from other components, is due to the recently discovered presence of probiotic bacteria in human milk. In addition to gastrointestinal benefits, modulation of microbiota by probiotic bacteria has been shown to regulate the immune function and to enhance defence against intestinal pathogens. Thus, the addition of breast milk probiotics to infant formulas could be a new alternative to mimic some of the functional effects of human milk in children who are not breastfed.

\section{Conflict of interest statement}

All the authors except JMR are employees at Puleva Biotech SA. All the studies presented have been funded by Puleva Biotech's own founds. This review has mainly been written by JX with collaboration of all the other authors.

\section{References}

1. Lopez-Alarcon M, Villalpando S \& Fajardo A (1997) Breastfeeding lowers the frequency and duration of acute respiratory infection and diarrhea in infants under six months of age. J Nutr 127, 436-443.
2. Wright AL, Bauer M, Naylor A, Sutcliffe E \& Clark L (1998) Increasing breastfeeding rates to reduce infant illness at the community level. Pediatrics 101, 837-844.

3. Saavedra JM (2002) Probiotic agents: clinical applications in infants and children. In Infant formula: closer to reference, pp. 15-27 [NCR Räihä and FF Rubaltelli, editors]. Philadelphia: Lippincott Williams \& Wilkins.

4. Isaacs CE (2005) Human milk inactivates pathogen individually, additively and sinergistically. $J$ Nutr 51, 1286-1288.

5. Hekkilä MP \& Saris PEJ (2003) Inhibition of Staphylococcus aureus by the commensal bacteria of human milk. J Appl Microbiol 95, 471-478.

6. Martin R, Langa S, Reviriego C, Jimenez E, Marin ML, Xaus J, Fernandez L \& Rodriguez JM (2003) Human milk is a source of lactic acid bacteria for the infant gut. $J$ Pediatr143, 754-758.

7. Ford JE, Law BA, Marshall VME \& Reiter B (1977) Influence of the heat treatment of human milk on some of its protective constituents. J Pediatr 91, 29-35.

8. Cesar JA, Victoria CG, Barros FC, et al. (1999) Impact of breast feeding on admission for pneumonia in postneonatal period in Brazil: nested case-control study. BMJ 318, 1316-1320.

9. Victoria CG, Smiyh PG, Vaughan JP, et al. (1987) Evidence for protection by breast-feeding against infant deaths from infectious diseases in Brazil. Lancet II 319-322.

10. Duncan B, Ey J, Holberg CJ, Wright AL, Martinez FD \& Taussing LM (1994) Exclusive breast-feeding for at least 4 months protects against otitis media. Pediatrics 91, 867-871.

11. Pisacane A, Graziano L, Mazzarella G, Scarpellino B \& Zona G (1992) Breast-feeding and urinary tract infections. J Pediatr 121, 331-332.

12. Silfverdal AS \& Olcen P (1999) Protective effect of breastfeeding: an ecologic study of Haemophilus influenzae meningitis and breastfeeding in a Swedish population. Int J Epidemiol 28, 152-156.

13. Grazioso CF, Werner AL, Alling DW, et al. (1997) Antiinflammatory effects of human milk on chemically induced colitis in rats. Pediatr Res 42, 639-643.

14. Garofalo RP \& Goldman AP (1999) Expression of functional immunomodulatory and antiinflammatory factors in human milk. Clin Perinatol 26, 361-367.

15. Hahn-Zoric M, Fulconis F, Minoli L, et al. (1990) Antibody response to parenteral and oral vaccines are impaired by conventional and low protein formulas as compared to breast-feeding. Acta Paediatr Scand 79, 1137-1142.

16. Harmsen HJM, Wildeboer-Veloo ACM, Raangs GC, et al. (2000) Analysis of intestinal flora development in breast-fed infants by using molecular identification and detection methods. J Pediatr Gstroenterol Nutr 30, 61-67.

17. Kunz C \& Rudloff S (1993) Biological functions of oligosaccharides in human milk. Acta Paediatr 82, 903-912.

18. Gilliland SE \& Speck ML (1977) Antagonistic action of lactobacillus acidophilus toward intestinal and food borne pathogens in associative cultures. $J$ food Prot 40, 820-823.

19. Conway PL (1996) Selection criteria for probiotic microorganisms. Asia pacific J Clin Nutr 5, 10-14.

20. Kalliomaki M, Salminen S, Arvilommi H, Kero P, Koskinen P \& Isolauri E (2001) Probiotics in primary prevention of atopic disease: a randomized placebo-controlled trial. Lancet 357, 1076-1079.

21. Dani C, Biandaioli R \& Firmito FR (2002) Potential role of probiotics in the prevention of necrotizing enterocolitis. In Infant formula: Closer to the reference. Philadelphia: Lippincott Williams \& Wilkins.

22. Gavin A \& Ostovr KMicrobiological characterization of human milk (1977) J Food Prot 40, 614-616.

23. Martin R, Olivares M, Marin ML, Fernandez L, Xaus J \& Rodriguez JM (2005) Probiotic potential of 3 lactobacilli strains isolated from breast milk. Hum Lact 21, 8-17. 
24. Martin R, Jimenez E, Olivares M, Marin ML, Fernandez L, Xaus J \& Rodriguez JM (2006) Lactobacillus salivarius CECT5713, a potential probiotic strain isolated from infant feces and breast milk of a mother-child pair. Int J Microbiol 112, 35-43.

25. Conway PL, Gorbach SL \& Goldin BR (1987) Survival of lactic acid bacteria in the human stomach and adhesion to intestinal cells. J Dairy Sci 70, 1-12.

26. Fons M, Gomez A \& Karjalainen T (2000) Mechanisms of colonization and colonization resistance of the digestive tract. Microb Ecol Health Dis 2, 240-246.

27. Olivares M, Diaz-Ropero MP, Martin R, Rodriguez JM \& Xaus J (2006) Antimicrobial potential of four lactobacillus strains isolated from breast milk. J Appl Microbiol 101, $72-79$.

28. Talarico TL \& Dobrogosz WJ (1989) Chemical characterization of an antimicrobial substance produced by Lactobacillus reuteri. Antimicrob Agents Chemother 33, 674-679.

29. Martin R, Olivares m, Marin ML, Xaus J, Fernandez L \& Rodriguez JM (2005) Characterization of a reuterin-producing Lactobcillus coryniformis strain isolated from a goat's milk cheese. Int J Food Microbiol 104, 267-277.

30. Diaz-Ropero MP, Martin R, Sierra S, Lara-Villoslada F, Rodriguez JM, Xaus J \& Olivares M (2006) Two Lactobacillus strains, isolated from breast milk, differently modulate the immune response. $J$ Appl Microbiol 102, 337-343.

31. Senok AC, Ismaeel AY \& Botta GA (2005) Probiotics: facts and myths. Clin Microb Infect 11, 958-966.

32. Björksten B, Naaber P, Sepp E \& Mikelsaar M (1999) The gut microflora in allergic Estonian and sweedish 2-year-old children. Clin Exp Allergy 29, 342-346.

33. Olivares M, Díaz-Ropero MP, Lara-Villoslada F, Rodriguez JM \& Xaus J (2005) Efectiveness of probiotics in allergy: child's game or adult affair? Nutrafoods 4, 59-64.
34. Peran L, Sierra S, Comalada M, et al. (2007) A comparative study of the preventative effects exerted by two probiotics, Lactobacillus reuteri and Lactobacillus fermentum, in the trinitrobenzenesulfonic acid model of rat colitis. Br J Nutr 97, 96-103.

35. Olivares M, Diaz-Ropero MP, Gomez N, Lara-Villoslada F, Sierra S, Maldonado JM, Martin R, Rodriguez JM \& Xaus J (2006) The consumption of two new probiotic strains, Lactobacillus gasseri CECT5714 and Lactobacillus coryniformis CECT5711, boost the immune system of healthy adults. Int Microbiol 9, 47-52.

36. Olivares M, Díaz-Ropero MP, Sierra S, Lara-Villoslada F, Fonolla J, Navas M, Rodriguez JM \& Xaus J (2007) Oral intake of Lactobacillus fermentum CECT5716 enhances the effect of influenza vaccination. Nutrition 23, 254-260.

37. Peran L, Camuesco D, Comalada M, Nieto A, Concha A, DiazRopero MP, Olivares M, Xaus J, Zarzuelo A \& Galvez J (2005) Preventative effects of a probiotic, Lactobacillus salivarius ssp salivarius, in the TNBS model of rat colitis. World J Gastroenterol 11, 5185-5192.

38. Olivares M, Díaz-Ropero MP, Gomez N, Lara-Villoslada F, Sierra S, Maldonado JA, Martin R, Lopez-Huertas E, Rodriguez JM \& Xaus J (2006) Oral administration of two probiotic strains, Lactobacillus gasseri CECT5714 and Lactobacillus coryniformis CECT5711, enhances the intestinal function of healthy adults. Int J Microbiol 107, 104-111.

39. Lara-Villoslada F, Sierra S, Boza J, Xaus J \& Olivares M (2007) Beneficial effects of consumption of a dairy product containing two probiotic strains, Lactobacillus coryniformis CECT5711 and Lactobacillus gasseri CECT5714n in healthy children. Nutr Hosp. 4, 22, 496-502.

40. Vendt N, Grünberg H, Tuure T, Malminiemi O, Wuolijoki E, Tillmann V, Sepp E \& Korpela R (2006) Growth during the first 6 months of life in infants using formula enriched with Lactobacillus rhamnosus GG: doble-blind, randomized trial. J Hum Nutr Dietet 19, 51-58. 